\title{
Research on Stochastic Resonance Detection Method for Periodic Signals under Low SNR and $\alpha$-Stable Noise
}

\author{
Zhaorui Li $\mathbb{D}^{1,2}$ Xiaobei Wu, ${ }^{1}$ Guangkai Liu $\mathbb{D}^{3},{ }^{3}$ Baofeng Guo $\mathbb{D}^{2},{ }^{2}$ and Bohang Chen ${ }^{2}{ }^{2}$ \\ ${ }^{1}$ School of Automation, Nanjing University of Science and Technology, Nanjing 210094, China \\ ${ }^{2}$ Department of Electronics and Optical Engineering, Army Engineering University Shijiazhuang Campus, \\ Shijiazhuang 050003, China \\ ${ }^{3}$ Beijing Institute of Tracking and Telecommunications Technology, Beijing 100094, China \\ Correspondence should be addressed to Baofeng Guo; guobao_feng870714@126.com
}

Received 2 January 2021; Revised 30 March 2021; Accepted 10 April 2021; Published 22 April 2021

Academic Editor: Peter Brida

Copyright (C) 2021 Zhaorui Li et al. This is an open access article distributed under the Creative Commons Attribution License, which permits unrestricted use, distribution, and reproduction in any medium, provided the original work is properly cited.

\begin{abstract}
In Dual Sequence Frequency Hopping (DSFH) communication mode, aiming at improving the detection performance to weak signal under low signal-to-noise ratio (SNR) conditions, stochastic resonance (SR) detection method is proposed. First, the $\alpha$-stable distribution is used as the impulsive noise model and the influence of $\alpha$ value on the properties of $\alpha$-stable noise is analyzed. Second, the transmitting and receiving signal model of DSFH communication system is introduced. The SR method is used to detect DSFH signal. In order to analyze the output signal, the fractional Fokker-Planck equation (FFPE) is established, and a new simplified solution method based on sampling decision time is proposed to solve the time-varying fractional differential equation. Base on the theoretical solution of FFPE, a binary hypothesis test statistic is constructed to quantify the signal detection probability and false alarm probability, and the detection performance is analyzed. Finally, simulation experiments verify the theoretical conclusions. The minimum effective SNR for SR detection is obtained, and it is about $-20 \mathrm{~dB}$, which provides a theoretical basis for the application of SR in the DSFH communication system.
\end{abstract}

\section{Introduction}

High frequency (HF)/very high frequency (VHF) hopping communication is the primary means in the campaign and tactical communication. In the HF and VHF band, there are many kinds of radiation sources, including communication, radar, navigation, identification of friend or foe (IFF), and other equipments. The electromagnetic environment is very bad. Under the condition of communication countermeasure, the communication signal will be seriously suppressed by the enemy's malicious interference. People have devoted themselves to research an emergency communication means suitable for low signal-to-noise ratio (SNR). Dual Sequence Frequency Hopping (DSFH) is a similar kind of communication method. The DSFH communication signal receiving can be attributed to the signal detection problem under very low SNR. Stochastic resonance (SR) method have been used to detect weak signals, but the noise background is often Gaussian white noise, which does not match the battlefield impulsive noise environment, resulting in large deviation of the research results. For DSFH communication mode, there is no research on the application of SR method for communication signal detection under impulse noise. The motivation of the research is to study an effective communication method for low SNR and impulsive noise.

SR detection of DSFH communication signal under impulse noise is the main content of this paper. DSFH is a kind of frequency hopping communication mode, based on "the medium is the message" [1]. DSFH was first proposed in $[2,3]$, the basic transmitting and receiving models were constructed, and its antijamming performance was analyzed. In the DSFH model, both the transmitter and the receiver have two channels which represent two states of 0 and 1 . Different from the traditional communication mode, the existence state of radio frequency (RF) sequences represents the symbols 0 and 1 . DSFH signal does not need to be 
demodulated, which reduces the quality requirements of the received signal. However, the antijamming ability is naturally stronger. Aiming at the poor antijamming ability of dual channels, linear frequency modulated (LFM) wave and pseudorandom code were modulated in DSFH RF signal to enhance the system's antijamming ability in [4-6].

Stochastic resonance (SR) theory is a powerful tool for weak signal detection under low SNR conditions. SR is applied to detect a weak harmonic signal or weak fault feature in [7-9]. The optimal local detector for the SR output based on the offset coefficient is studied in [10]. The detection of BPAM, BFSK, and BPSK signal using SR is realized in $[11,12]$. In the DSFH communication mode, the feasibility of SR is demonstrated and the quantitative results to a suitable range of SNR and BER are given in $[13,14]$.

The SR principle is that the weak signal uses noise energy drive particles to resonance between two potential wells. Its essence is a kind of energy transfer from noise to weak signal, which increases the SNR. Therefore, noise is an essential condition for SR system, and selecting a suitable noise model is the key to study DSFH communication performance. Gaussian white noise is used in classical SR, and many researchers study SR under Gaussian white noise [15-18]. Although the Gaussian distribution has a solid theoretical foundation and mature application, many investigations have shown that it is not suitable to describe impulsive noise. This mismatch will lead to performance degradation or failure of the signal detection algorithm.

Battlefield wireless communication environment contains many random intense pulses, which show typical nonGaussian property. The common non-Gaussian mathematical models mainly include Gaussian mixture distribution, t-distribution, generalized Gaussian distribution, and $\alpha$-stable distribution. Alpha-stable distribution is a typical thick-tailed distribution, which is successfully used in modeling atmospheric and marine noise, and it is also widely used in other impulsive noise situations. It has a complete mathematical definition and characteristic function. It is convenient for theoretical analysis and closely related to the statistical physical model [19-23]. Therefore, in this paper, $\alpha$-stable distribution is used as the impulsive noise model, so SR detection is studied under $\alpha$-stable noise.

There are two main ways to study SR. One is the signal simulation method based on the Langevin equation (LE), the other is the theoretical method by solving probability density function (PDF) based on Fokker-Planck equations (FPE), and the SR output signal is analyzed based on the solution.

In $[24,25]$, the solution process of fractional FokkerPlanck Equations (FFPE) is studied and the stationary solution of the SR system is obtained by the approximate method. The path integral solution (PIS) is adopted for determining the PDF and mean first-passage time (MFPT) in $[26,27]$. The bistable system SR phenomenon with the periodic input signal and $\alpha$-stable noise is demonstrated by the numerical simulation method in [28]. The SR phenomenon under $\alpha$-stable noise is verified, and the bistable system's response excited by constant signal and periodic square wave signal are studied in [29-31]. The parameters induced SR are studied and the relationship between system parameters $a, b$, and SNR with $\alpha$-stable noise is analyzed by simulation experiments in [30]. In recent years, many domestic and foreign scholars have utilized different potential functions to realize the SR detection under $\alpha$-stable noise [32-37]. The problem of weak signal detection using a cascade SR system is studied in [38]. The study of SR in $\alpha$-stable noise has attracted more and more attention [39-44].

In this paper, the SR method is applied to detect DSFH signal under impulsive noise, and a new simplified solution method based on sampling decision time is proposed to solve the time-varying fractional differential equation. The accuracy of theoretical solution is demonstrated by simulation experiments, and some quantitative performances are obtained for the first time. By analyzing parameter correlation between bistable system, periodic signal and noise, the influence of parameter changes on SNR, suitable SNR range, and achievable BER can be obtained quantitatively. The detection probability and false alarm probability can be described qualitatively.

\section{Alpha-Stable Noise}

2.1. Definition and Mathematical Expression. $\alpha$-stable distribution is a mathematical empirical model suitable for impulsive noise modeling. It satisfies the generalized central limit theorem. The sum of infinite independent and identically distributed random variables is $\alpha$-stable distribution. Unlike the central limit theorem, the random variables may not be finite variance, which makes it more widely used. Alpha-stable distribution does not have a unified PDF, but it has a characteristic function as follows:

$$
\varphi(t)=\exp \left\{j \mu t-\gamma|t|^{\alpha}[1+j \beta \operatorname{sign}(t) \omega(t, \alpha)]\right\},
$$

where $\quad \omega(t, \alpha)=\left\{\begin{array}{ll}\tan (\alpha \pi / 2), & \alpha \neq 1, \\ (2 / \pi) \log |t|, & \alpha=1,\end{array} \quad\right.$ and $\quad \operatorname{sign}(t)=$ $\begin{cases}1, & t>0, \\ 0, & t=0,\end{cases}$ $-1, \quad t<0$.

described by adjusting its parameters.

$\alpha(0<\alpha \leq 2)$ is the characteristic parameter, which determines the impulsivity of $\alpha$-stable distribution. The smaller the value of $\alpha$ is, the stronger the impulsivity is, the higher the probability of the abnormal value is, and the thicker the tail is in PDF. Since $\alpha$-stable distribution turns into Gaussian distribution when $\alpha=2, \alpha$-stable distribution is a generalized form of Gaussian distribution.

$\beta(-1 \leq \beta \leq 1)$ is the skew parameter which determines the skewness of $\alpha$-stable distribution. When $\beta=0$, it is called symmetric $\alpha$ stable $(S \alpha S)$ distribution.

$\gamma(\gamma>0)$ is the scale parameter, which is used to describe the random variables dispersion from the mean or median value.

$\mu$ is the shift parameter, which is used to describe the position of the PDF of $\alpha$-stable distribution. When $\mu=0$ and $\gamma=1$, it is called the standard $\alpha$ stable distribution. When $\alpha=2$ and $\beta=0$, the $\alpha$-stable distribution becomes Gaussian distribution. When $\alpha=1$ and $\beta=0$, the $\alpha$-stable distribution becomes Cauchy distribution. When $\alpha=1 / 2$ and $\beta=1$, the $\alpha$-stable distribution becomes Levy distribution. 
2.2. Generation of $\alpha$-Stable Distribution Random Variables. Simulation is a main mean to study $\alpha$-stable noise and signal processing in the noise. The generation of $\alpha$-stable distribution random variables is an important condition. If $\xi$ is $\alpha$-stable distribution random numbers, which can be generated by the Janicki Weron (JW) algorithm [24]:

$$
\begin{aligned}
\xi= & D_{\alpha, \beta, \gamma} \frac{\sin \left(\alpha\left(V+C_{\alpha, \beta}\right)\right)}{(\cos (V))^{1 / \alpha}} \times\left[\frac{\cos \left(V-\alpha\left(V+C_{\alpha, \beta}\right)\right)}{W}\right]^{(1-\alpha) \alpha} \\
& +\mu
\end{aligned}
$$

where $\quad C_{\alpha, \beta}=(\arctan (\beta \tan (\pi \alpha / 2)) / \alpha), \quad D_{\alpha, \beta, \gamma}=\gamma[\cos$ $(\arctan (\beta \tan (\pi \alpha / 2)))]^{-1 / \alpha}, V$ is a random variable uniform distributed over $(-\pi / 2, \pi / 2)$, and $W$ is a random variable exponentially distributed with a unit mean. The PDF curve of $\alpha$-stable distribution with a different $\alpha$ is shown in Figure 1. It can be seen that the smaller $\alpha$ is, the stronger the impulsivity is.

\section{Theoretical Analysis of SR Signal Detection in DSFH Communication System}

\subsection{Analysis of DSFH Receiving Signal Property}

3.1.1. The Transmitting Signal Model of DSFH. In DSFH model, when sending data "0," channel 0 is used as the data channel to transmit frequency signal, and channel 1 as the dual channel does not transmit frequency signal, as shown in Figure 2.

The carrier frequency of channel 0 is $f_{0, n}$ which is controlled by pseudorandom (PN) sequence $\mathrm{FS}_{0}$, and the carrier frequency of channel 1 is $f_{1, n}$ which is controlled by the $\mathrm{PN}$ sequence $\mathrm{FS}_{1}$. When the transmitting symbol is 0 , the carrier frequency $f_{0, n}$ controlled by the PN sequence $\mathrm{FS}_{0}$ is transmitted. When the transmitting symbol is 1 , the carrier frequency $f_{1, n}$ controlled by the PN sequence $\mathrm{FS}_{1}$ is transmitted. At the time of $t$, the sinusoidal signal $s_{0}(t)$ with the frequency of $f_{0, n}$ is transmitted when the transmitted symbol is 0 . Otherwise, the sinusoidal signal $s_{1}(t)$ with the frequency of $f_{0, n}$ is transmitted when the transmitted symbol is 1 . Moreover, the signals $s_{0}(t)$ and $s_{1}(t)$ are both sinusoidal. After the channel switch, the final transmitted signal $s(t)$ of DSFH is the combination of $s_{0}(t)$ and $s_{1}(t)$.

Assume that the transmitted data is $(\ldots, 1,0,1,0, \ldots)$, for the main analysis, and the baseband filter is neglected. Then, the transmitted signal is the sine carrier with a frequency of $\left(\ldots, f_{2}, f_{4}, f_{3}, f_{1}, \ldots\right)$, and the RF signal of DSFH can be represented as

$$
\begin{aligned}
s(t)= & \cos \left[2 \pi f_{i, n}\left(t-n T_{s}\right)+\varphi\right] \\
& \cdot\left[\varepsilon\left(t-n T_{s}\right)-\varepsilon\left(t-(n+1) T_{s}\right)\right],
\end{aligned}
$$

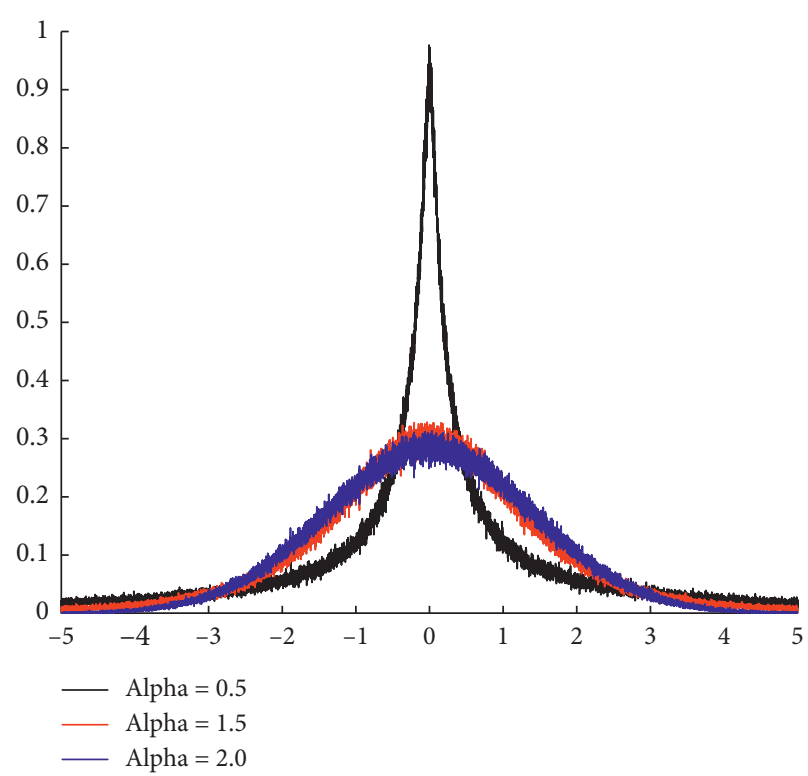

FIgURE 1: PDF of $\alpha$-stable distribution with a different $\alpha$.

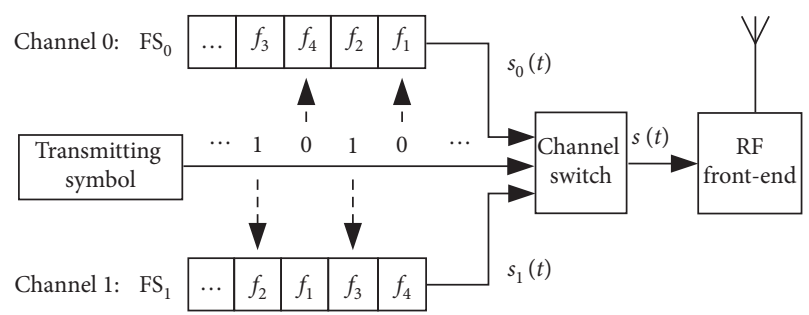

FIGURE 2: The transmitting structure of DSFH.

where $T_{s}$ is the hop duration, $\varepsilon(t)$ is the step signal, $f_{i, n}$ is the RF of the $n$th hop, and $f_{i, n}=$ $\left\{\begin{array}{ll}f_{0, n}, & \text { transimtting symbol is } 0, \\ f_{1, n}, & \text { transimtting symbol is } 1,\end{array}\right.$ and $f_{0, n} \neq f_{1, n}$.

3.1.2. The Receiving Signal Model of DSFH. The superheterodyne receiver is adopted in DSFH mode, which is depicted in Figure 3. The receiver receives the signal of two channels according to the same frequency hopping pattern of the transmitter. After receiving the signal $r(t)$, the RF front-end carries out mixing and band-pass filtering with the carrier signal controlled by the corresponding PN sequence. The waveform and frequency of the intermediate frequency (IF) signal obtained by the two branches are the same, which can be shown as

$$
\begin{aligned}
s(t)= & \cos \left[2 \pi f_{0}\left(t-n T_{s}\right)+\varphi\right] \\
& \cdot\left[\varepsilon\left(t-n T_{s}\right)-\varepsilon\left(t-(n+1) T_{s}\right)\right],
\end{aligned}
$$

where $f_{0}$ is the preset frequency of the IF signal.

By analyzing the DSFH model, we can see that the received signal after frequency mixing is a single frequency 


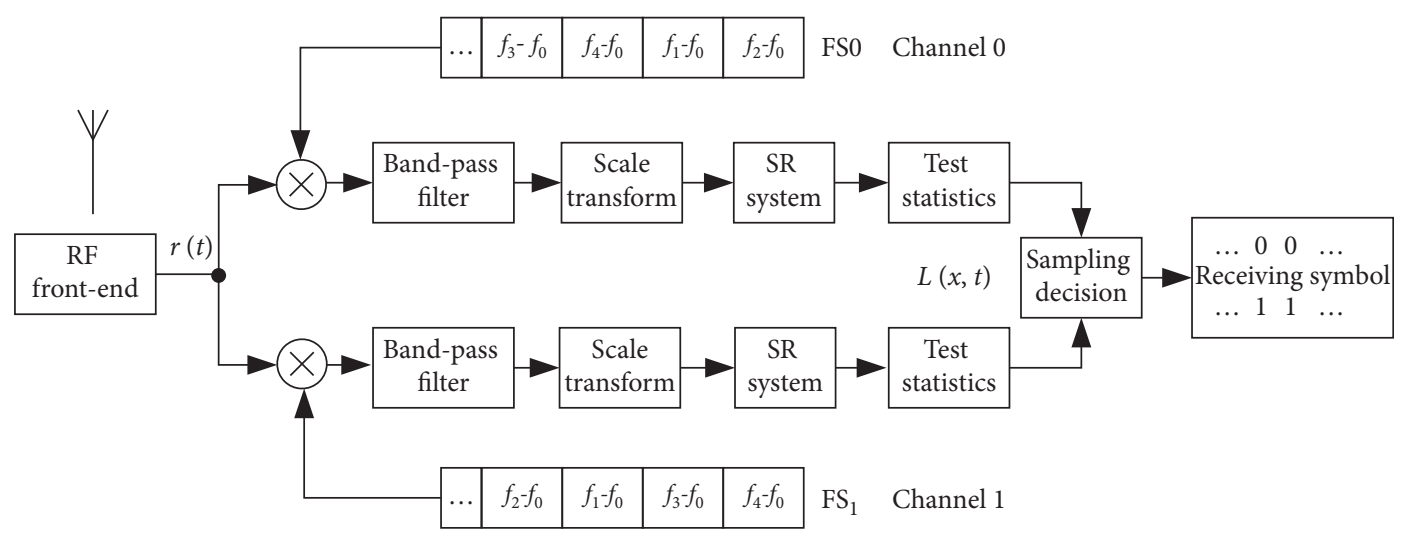

Figure 3: The receiving structure of the DSFH.

sinusoidal signal. So, the problem of DSFH signal receiving becomes the problem of judging whether the sinusoidal signal exists.

\subsection{Theoretical Analysis of SR Signal Detection under $\alpha$-Stable} Noise. Driven by the $\alpha$-stable noise and periodic signal, the output of the bistable SR system can be described by LE, which is shown as

$$
\frac{\mathrm{d} x}{\mathrm{~d} t}=a x-b x^{3}+s(t)+\eta(t),
$$

where $s(t)$ is a weak external sinusoidal signal and $\eta(t)$ is asymmetric unbiased $\alpha$ stable distribution white noise with characteristic parameter $\alpha$ and noise intensity $D$. In this paper, zero-skewness and zero-shift will be considered, so $\beta=0, \mu=0$, and according to formula (6), $D=\gamma$. A typical nonlinear potential system is utilized in the bistable system as

$$
U(x)=-\frac{a}{2} x^{2}+\frac{b}{4} x^{4}, \quad a>0, b>0 .
$$

LE describes amplitude property of the output signal. The FFPE corresponding to the statistical property of the output signal is shown as

$$
\begin{aligned}
\frac{\partial}{\partial t} \rho(x, t)= & \frac{\partial}{\partial x}\left\{\left[-a x+b x^{3}-s(t)\right] \rho(x, t)\right\} \\
& +D \frac{\partial^{\alpha}}{\partial|x|^{\alpha}} \rho(x, t),
\end{aligned}
$$

where $\rho(x, t)$ is the PDF of the output signal, which represents the amplitude statistical property at time $t . s(t)$ is assumed to be expressed as $A \cos (\omega t+\varphi)$, and formula (7) contains a time-varying term without stationary solution. Since the signal transformation is instantaneous, it can be assumed that the signal reaches a stable state instantaneously and no longer changes with time as $(\partial / \partial t) \rho(x, t)=0$. The sampling value on signal decision time $t_{0}$ in (7) can be expressed as

$$
\frac{\partial}{\partial x}\left\{\left[-a x+b x^{3}-s\left(t_{0}\right)\right] \rho\left(x, t_{0}\right)\right\}+D \frac{\partial^{\alpha}}{\partial|x|^{\alpha}} \rho\left(x, t_{0}\right)=0,
$$

where $s\left(t_{0}\right)$ is regarded as a constant $s_{0}$, and (8) can be rewritten as

$$
\frac{\partial}{\partial x}\left\{\left[-a x+b x^{3}-s_{0}\right] \rho(x)\right\}+D \frac{\partial^{\alpha}}{\partial|x|^{\alpha}} \rho(x)=0,
$$

where $a, b, s_{0}$, and $D$ are constants.

The SR output signal $\rho(x)$ can be obtained and analyzed by solving the fractional differential equation in (9). In this paper, we use finite difference method to solve it (9).

According to the definition of Gyunwald-Letnikov (GL) fractional calculus, after the differential processing, the equation is

$$
\begin{gathered}
\left(-a+3 b x_{i}^{2}\right) \rho\left(x_{i}\right)+\left(-a x_{i}+b x_{i}^{3}-s_{0}\right) \frac{\rho_{i}-\rho_{i-1}}{h} \\
+\frac{D}{h^{\alpha}} \sum_{k=0}^{i+1} g_{k} \rho_{i-k+1}+\frac{D}{h^{\alpha}} \sum_{k=0}^{M-i+1} g_{k} \rho_{i+k-1}=0
\end{gathered}
$$

where $g_{k}=(-1)^{k}\left(\begin{array}{l}\alpha \\ k\end{array}\right),\left(\begin{array}{l}\alpha \\ k\end{array}\right)=(\alpha(\alpha-1) \cdots(\alpha-k+1) /$ $k !), h=(R-L / M)$, and $R$ and $L$ are left and right bounds of the signal amplitude boundary, respectively. The $R$ and $L$ space domain is divided into $M$ divisions in the differential processing.

Let $p_{i}=\left(-a+3 b x_{i}^{2}\right), 0 \leq i \leq M$ and $q_{i}=\left(-a x_{i}+b x_{i}^{3}-s_{0}\right)$, $0 \leq i \leq M$, with

$$
\begin{gathered}
{\left[p_{i}+\frac{q_{i}}{h}+\frac{2 D g_{1}}{h^{\alpha}}\right] \rho_{i}+\left[-\frac{q_{i}}{h}+\frac{D g_{2}}{h^{\alpha}}+\frac{D g_{0}}{h^{\alpha}}\right] \rho_{i-1}} \\
+\left[\frac{D g_{2}}{h^{\alpha}}+\frac{D g_{0}}{h^{\alpha}}\right] \rho_{i+1} \\
+\frac{D}{h^{\alpha}} \sum_{k=3}^{i+1} g_{k} \rho_{i-k+1}+\frac{D}{h^{\alpha}} \sum_{k=3}^{M-i+1} g_{k} \rho_{i+k-1}=0 .
\end{gathered}
$$

Let $\rho=\left[\rho_{1}, \rho_{2}, \cdots, \rho_{M-1}\right]^{T}$, and $A_{i j}$ is a matrix with the size of $(M-1) \times(M-1)$, where 


$$
A_{i j}= \begin{cases}p_{i}+q_{i}+\frac{2 D g_{1}}{h^{\alpha}}, & j=i, \\ -q_{i}+\frac{D g_{2}}{h^{\alpha}}+\frac{D g_{0}}{h^{\alpha}}, & j=i-1, \\ \frac{D g_{2}}{h^{\alpha}}+\frac{D g_{0}}{h^{\alpha}}, & j=i+1, \\ \frac{D}{h^{\alpha}} g_{i-j+1}, & j \leq i-2, \\ \frac{D}{h^{\alpha}} g_{j-i+1}, & j \geq i+2,\end{cases}
$$

with

$$
A_{i j} \rho=0 \text {, }
$$

where $\rho_{0}=0, \rho_{M}=0$, and $\sum_{i=1}^{M-1} h \rho_{i}=1$.

The Gauss elimination method is used to obtain the solution of equation (13), which is also the probability density of SR output signal. The PDF without weak sinusoidal signal input can be also obtained by the same method. The difference between the two states can be analyzed theoretically.

Due to the bistable system's effect, the PDF has changed from a single peak structure to an asymmetric bimodal structure, indicating that particles gather in two potential wells. The SNR was calculated and SNR peak appears obviously. It proves the occurrence of SR phenomenon. With sinusoidal signal driving, the peak value is higher than that without driving, which indicates that the weak periodic force affects particles' aggregation. The difference is amplified by the SR system, which proves the effectiveness of SR to detect weak sinusoidal signal.

3.3. Performance Analysis of Detection and Reception. In the DSFH system, two channels receive signals separately. Since the two channels cannot receive two signals at the same time, signal reception can be considered as binary hypothesis test problem.

$$
\left\{\begin{array}{l}
H_{0}: x\left(t_{0}\right)=x_{0}\left(t_{0}\right), \\
H_{1}: x\left(t_{0}\right)=x_{1}\left(t_{0}\right),
\end{array}\right.
$$

where $x_{0}\left(t_{0}\right)$ is SR output driven by only impulsive noise, $x_{1}\left(t_{0}\right)$ is the SR output driven by weak sinusoidal signal and impulsive noise of DSFH system received, and $t_{0}$ is the sampling decision time. Based on Bayesian criterion, maximum a posterior probability (MAP) can be used for binary communication problems, and the test statistic can be obtained as

$$
\Lambda\left(x, t_{0}\right) \stackrel{H_{1}}{\gtrless} \eta,
$$

where $\eta$ is the decision threshold and $\Lambda\left(x, t_{0}\right)$ is expressed as

$$
\Lambda\left(x, t_{0}\right)=\frac{P\left(x \mid H_{1}, t_{0}\right)}{P\left(x \mid H_{0}, t_{0}\right)} .
$$

The detection probability $P_{d}$ is the probability that the real state is $H_{1}$ and it is judged as $H_{1}$. The false alarm probability $P_{f a}$ is the probability that the state is $H_{0}$, but it is judged as $H_{1}$. The PDF with or without periodic signal, which has been solved by theoretical method, is shown in Figure 4. The decision area and decision probability of SR is marked, where $r$ is the decision threshold.

Since the motion orbit of the SR particles is symmetrical, the PDF of the SR system output signal and the decision domain are also symmetrical. The decision domain of $H_{1}$ is $R_{1}=\{R|| R \mid>\eta\}$. The decision domain of $H_{0}$ is $R_{0}=\{R|| R \mid$ $>\eta\}$.

$P_{d}$ can be obtained as

$$
P_{d}=\int_{R_{1}} P\left(x \mid H_{1}\right) \mathrm{d} x .
$$

$P_{f a}$ can be obtained as

$$
P_{f a}=\int_{R_{1}} P\left(x \mid H_{0}\right) \mathrm{d} x .
$$

Based on the PDF of the SR system output signal, we can obtain $P_{d}$ and $P_{f a}$.

\section{Simulation Results and Discussion}

To the typical bistable system represented by equation (6), according to the adiabatic approximation theory, there are two identical potential wells and one potential barrier. The potential well position is located at $x_{m}= \pm \sqrt{a / b}$, the barrier center is at 0 , and the barrier height is $\Delta U=a^{2} / 4 b$. When there is only noise and no sinusoidal signal excitation, the particles will vibrate in the potential wells with small noise, while the particles will transition between the two potential wells according to Kramers escape rate when the noise increases, and the transition is random with no periodicity. However, SR will occur in the system if the sinusoidal signal is added, and the transition frequency of the particles is the same as the sinusoidal signal.

4.1. Verification of SR Phenomenon. For the bistable system in equation (6), based on the requirements of a low-frequency small signal for the adiabatic approximation theory, sine wave is selected as the periodic input signal. The frequency of the sinusoidal signal is $10 \mathrm{~Hz}$, and the amplitude is 0.1 . The signal is in the $\alpha$-stable noise with $\alpha=1.5, D=0.3$, and $\mathrm{SNR}=-15 \mathrm{~dB}$. The sampling frequency is $200 \mathrm{KHz}$. To increase the adaptability and dynamic of the bistable system, let $a=10000$, and $b$ ranges from $1 e^{10}$ to $1 e^{13}$. The simulation results is shown in Figure 5(a), where the vertical axis is the output SNR, and the horizontal axis is the parameter $b$, and the actual value is $b \times e^{10}$. It can be seen that when $b$ is higher than $4 e^{12}$, the SNR reaches the maximum region. 


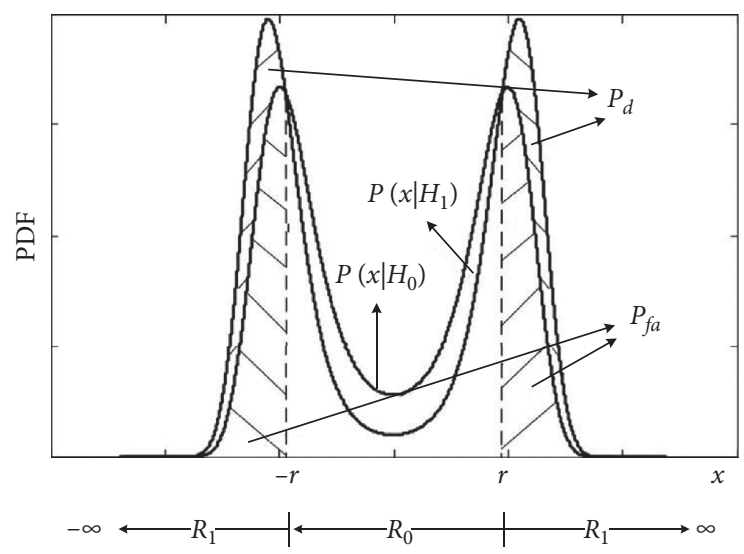

FIGURE 4: Decision areas of detection probability and false alarm probability.

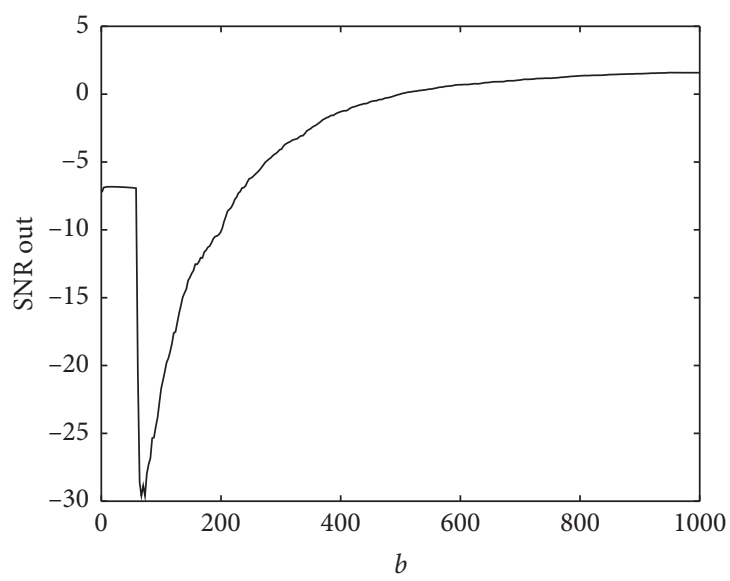

(a)

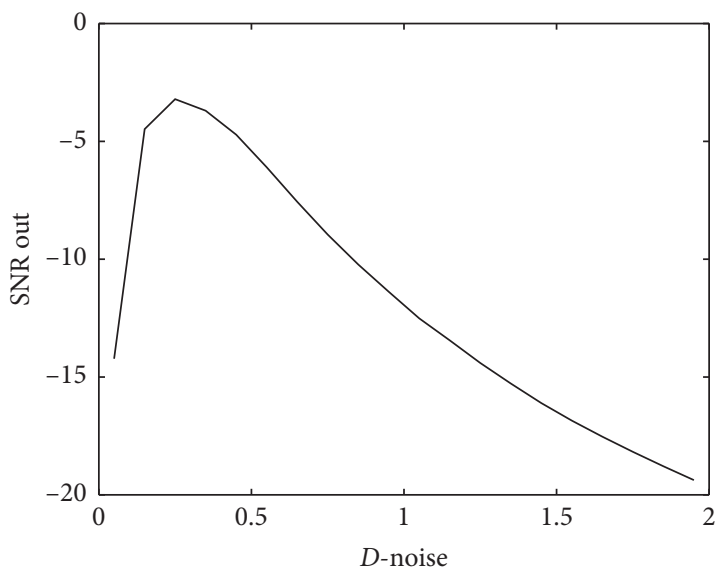

(b)

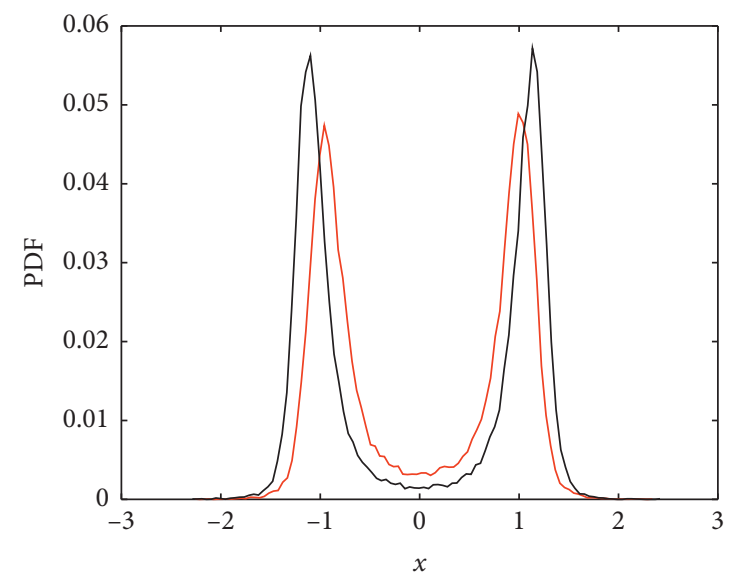

(c)

FIGURE 5: Parameter $b$ optimization and SR phenomenon verification: (a) $f=10 \mathrm{~Hz}, \alpha=1.5, D=0.3$, and SNR $=-15 \mathrm{~dB}, a=10000$, and parameter $b$ varies from $1 e^{10}$ to $1 e^{13}$, the change of output SNR; (b) $a=10000, b=4.2 e^{12}$, and noise intensity $D$ varies from 0.01 to 2 , the change of output SNR curve; (c) PDF of SR output with and without sinusoidal signal. Red means no signal is added.

Let $a=10000$ and $b=4.2 e^{12}$, and the simulation results are shown in Figure 5(b), where the vertical axis is SNR, and the horizontal axis is the noise intensity $D$. The amplitude of the input period weak signal is 0.1 . The output SNR increases with the increase of noise intensity obviously, and the peak value appears when $D$ is about 0.3 . It indicates that the energy of the noise transfers to the signal during the process, and the SR effect is better near $D=0.3$. The adiabatic 
approximation theory gives an analytical expression for the relationship between noise intensity and SNR. There is no analytical expression for impulsive noise, but the phenomenon is the same as SR under Gaussian noise. The results are the same as the previous theoretical calculation results. Figure 5(c) shows the probability density curve of the output signal. Red indicates the output without sinusoidal signal, and black represents the output after adding sinusoidal signal. It can be seen that the signal probability density is more concentrated after adding the signal, and the difference is more obvious.

The SR phenomenon can also be proved by the timedomain output signal of the bistable system. As shown in Figure 6, the first line shows the input signal, the second line shows the output signal of the SR system, and the third line shows the periodic input signal. As shown in Figure 6(a), in the case of no sinusoidal signal input, although the output signal of the bistable system is also two-state switching, the periodicity cannot be seen. As shown in Figure 6(b), after adding a sin signal with amplitude of 0.1 , the periodic property of the input signal cannot be seen because the signal is covered by noise. However, the periodicity of the output is more obvious after the SR system, which also proves the SR phenomenon.

Figure 7 shows the PDF of the bistable system output signal under different noise intensities. Red is the SR output without a sinusoidal signal, and black is the output after adding a sinusoidal signal. Figure 7 (a) shows the PDF of the bistable system output signal when the noise intensity is 0.1 . Due to the low noise intensity, the system does not have a potential well transition, and the output signal is mainly in a single well. When the sinusoidal signal is added, the SR phenomenon occurs. Figure 7(b) shows the PDF of the bistable system output signal when the noise intensity is 0.15 . There is no sinusoidal signal input in the system, but the distribution of the two potential wells is not uniform. Increasing the noise intensity further, as shown in Figure $7(\mathrm{c})$, the transition between two potential wells can occur with or without sinusoidal signal, but SR only occurs when sinusoidal signal input.

4.2. Influence of Noise Parameter $\alpha$ on SR. Alpha is an important parameter of $\alpha$-stable distribution, which determines the impulsivity of noise. The smaller $\alpha$ is, the stronger the impulse and the thicker the tail of PDF will be, which indicates that there are more abnormal values. Different impulse degrees will have a significant impact on the signal reception, especially the SR. The following experiment mainly focuses on the influence of $\alpha$ on SR.

First, the stronger the impulse of noise, the more likely it is to cause SR. As shown in Figures 8(a)-8(d), there are the input and output SNR curves of the SR system when the noise parameter $\alpha$ is $2.0,1.5,0.85$, and 0.5 , respectively. The red line is the input SNR curve calculated according to the $10 \mathrm{~Hz}$ sinusoidal signal and noise amplitude in time domain. The blue line is the mixed signal and noise SNR curve calculated by FFT in frequency domain. It can be seen that the SNR curves of the two methods are the same. The black line is the SNR curve of SR output signal. Compared with the FFT method, SR can generate SNR gain, which is about $22 \mathrm{~dB}, 19 \mathrm{~dB}, 17 \mathrm{~dB}$, and $14 \mathrm{~dB}$. It indicates that SR occurs, and the stronger the impulsivity is, the smaller the gain is. However, in the SR stage, the stronger the noise impulsivity is, the more likely it is to induce SR. When the noise intensity is $0.5,0.25,0.02$, and near-zero, the SNR output peak is reached. It shows that the larger the abnormal value of the noise, the easier the energy transfer and the more likely to generate SR.

Under $\alpha$-stable noise, the theoretical and simulation values of PDF after SR processing to the same SNR and a different $\alpha$ are shown in Figure 9. With the same SNR, it can be seen that the smaller the $\alpha$ is, the more different the PDF of SR output signal is. The reason is that $\alpha$ can be used to characterize the impulsivity of $\alpha$-stable noise. The smaller the $\alpha$ is, the stronger the color property of noise is. The large the difference between $\alpha$-stable distribution and Gaussian white noise is, the more favorable it is for SR to start resonance and achieve the best resonance state and the better the resonance effect is. When $\alpha=2, \alpha$-stable noise becomes Gaussian white noise, and the PDF difference is the smallest. It is proved that the driving impact of Gaussian white noise on SR is worse than that of $\alpha$-stable noise, that is, color noise is more conducive to SR.

4.3. Influence of Input SNR to SR. Under $\alpha$-stable noise, the theoretical and simulation value of PDF after SR processing to the same $\alpha$ and different SNR are shown in Figure 10. It can be seen that when $\alpha$ is the same, the larger the SNR is, the greater the sinusoidal signal amplitude and the driving force are, which means the better the starting resonance and SR effect. Under $\alpha$-stable noise, the difference of output PDF with or without sinusoidal signal makes it possible for signal detection and processing.

4.4. Analysis of System Receiving Performance. $P_{d}$ and $P_{f a}$ can be obtained based on PDF. The receiver operation characteristic (ROC) curve of sinusoidal signal after SR processing in $\alpha$-stable noise is shown in Figure 11, reflecting the relationship between $P_{d}$ and $P_{f a}$. A different $\alpha$ corresponds to different ROC curves when $\mathrm{SNR}=-8 \mathrm{~dB}$. Regardless of the $\alpha$ value of $0.5,1.5$, or 2.0 , the ROC curve is far above the $P_{d}=P_{f a}$ line, which represents good detection performance.

The BER can be obtained based on $P_{d}$ and $P_{f a}$. The BER curve of DSFH sinusoidal signal with $\alpha$-stable noise after SR processing is shown in Figure 12. It can be seen that the theoretical value and simulation value of $P_{e}$ decrease with the increase of SNR. It is because the improvement of SNR can effectively improve the detection probability. Meanwhile, the smaller the $\alpha$ is, the smaller the $P_{e}$ is. The reason is that the noise power is constant when the SNR is fixed, and the smaller $\alpha$ represents the more substantial noise impact, which is more conducive to electromagnetic particles in SR potential wells. Therefore, the detection performance is better. It can be proved that the DSFH signal can be detected 


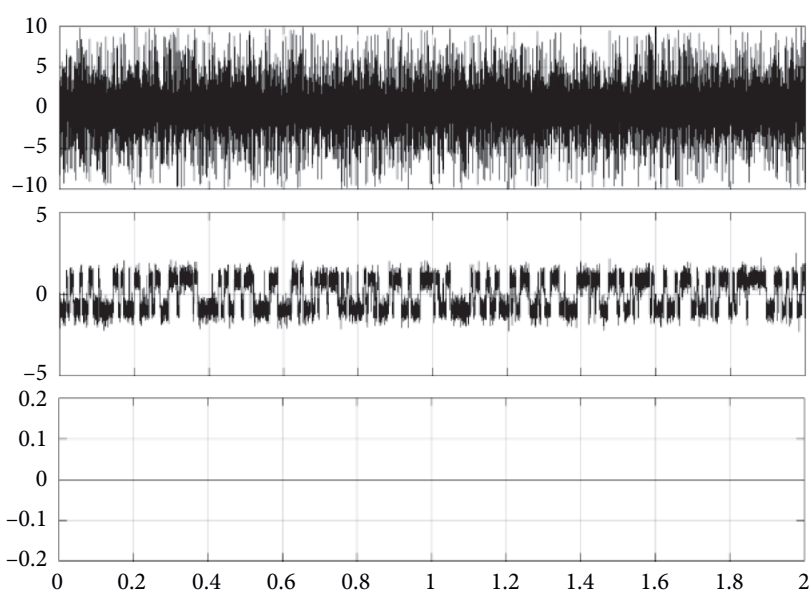

(a)
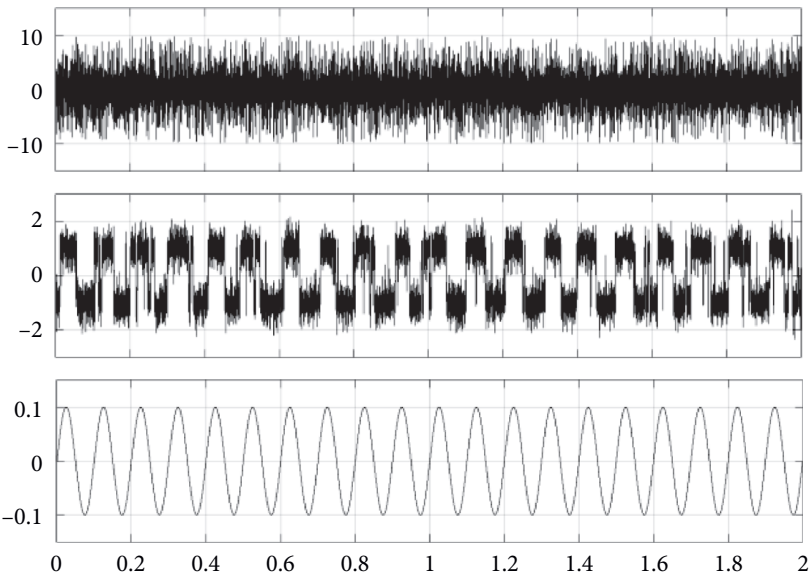

(b)

FIgURE 6: Comparison diagram of SR system input and output signal. (a) Without sinusoidal signal. (b) With sinusoidal signal.

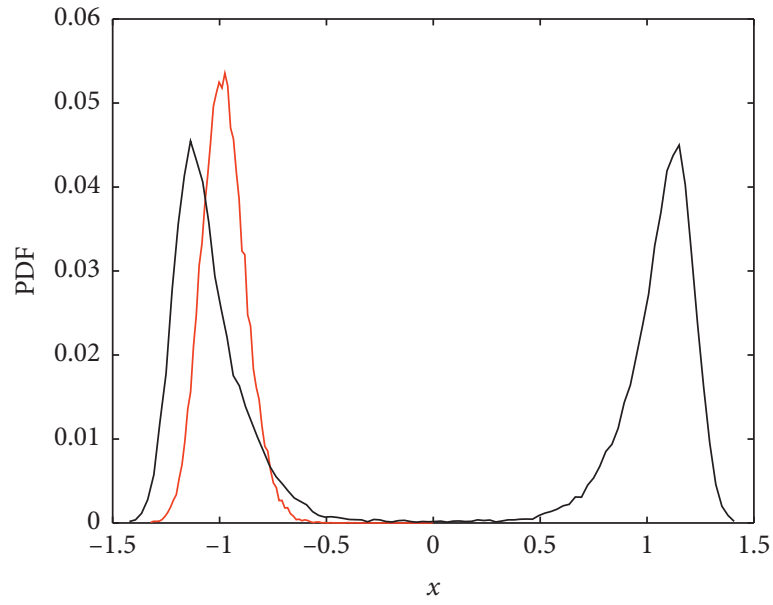

(a)

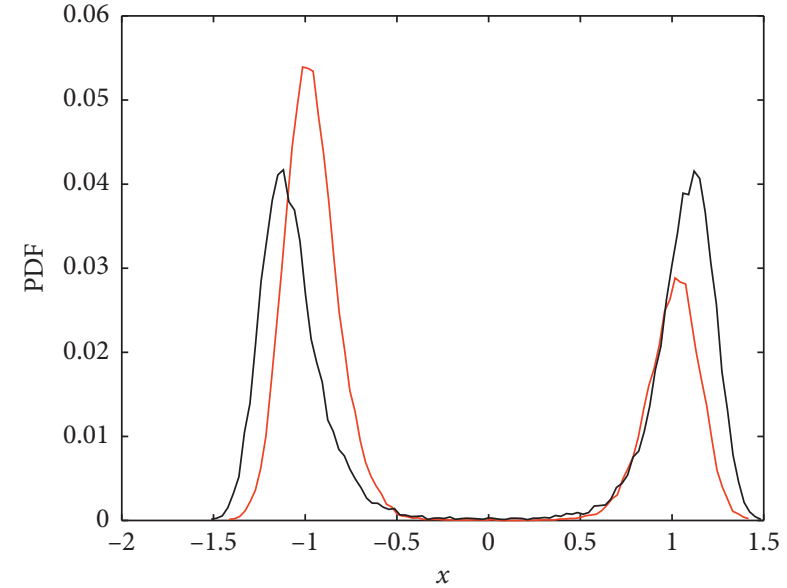

(b)

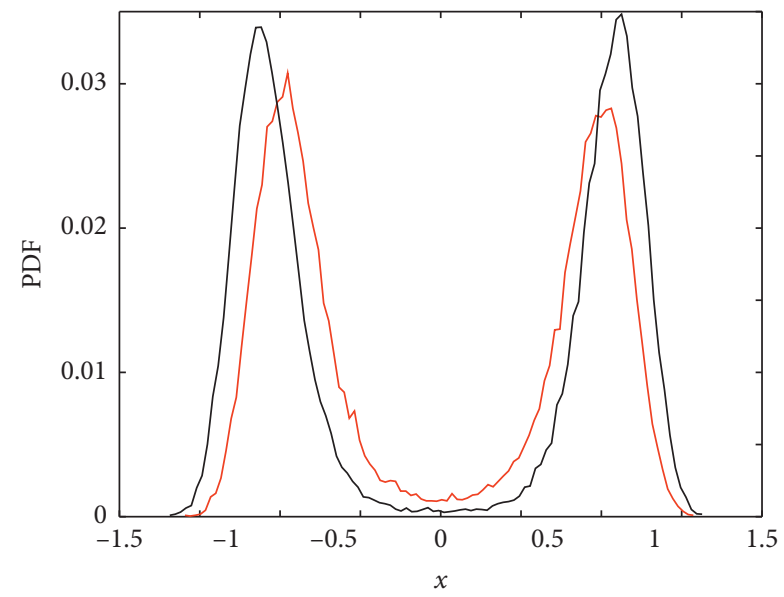

(c)

FIGURE 7: Diagram of SR occurrence process, $f=10 \mathrm{~Hz}, \alpha=1.5, A=0.1, a=10000$, and $b=4.2 * e^{12}$, and the influence of $D$ on SR output. (a) Noise intensity is 0.15 . (b) Noise intensity is 0.25 . (c) Noise intensity is 0.3 . 


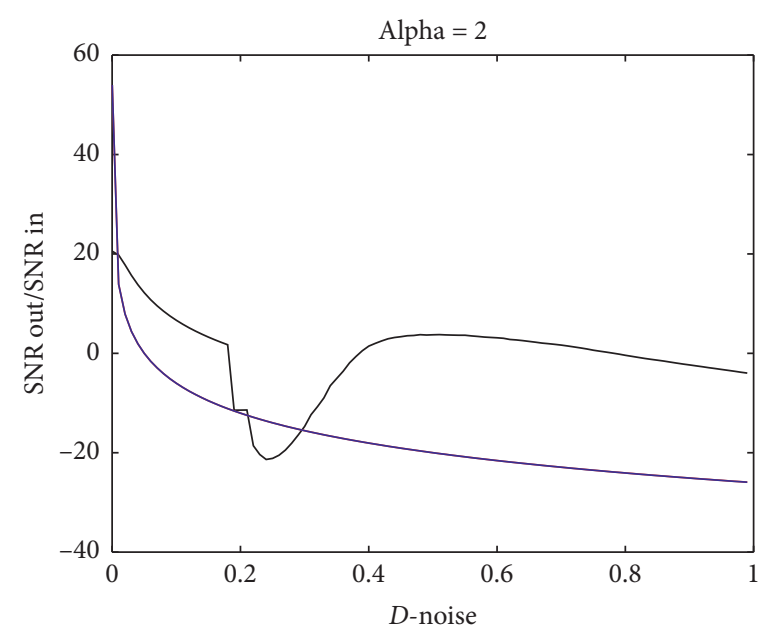

(a)

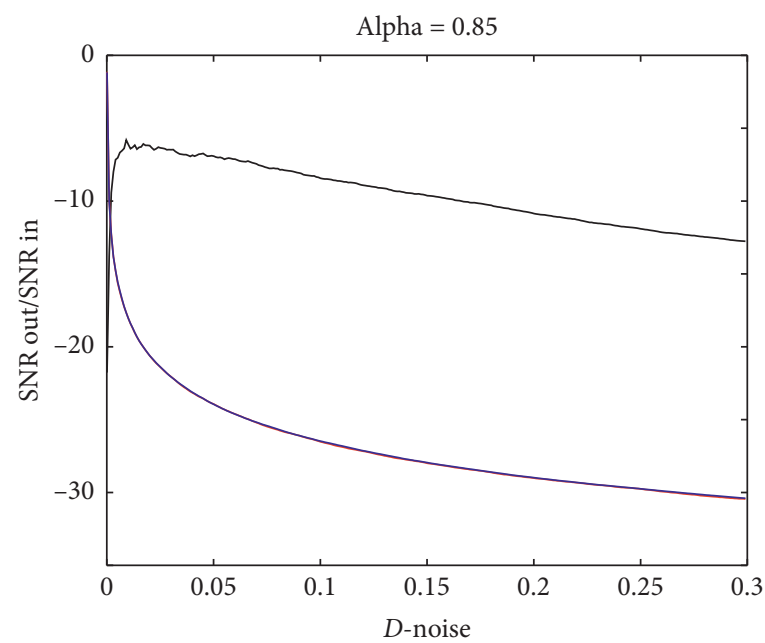

(c)

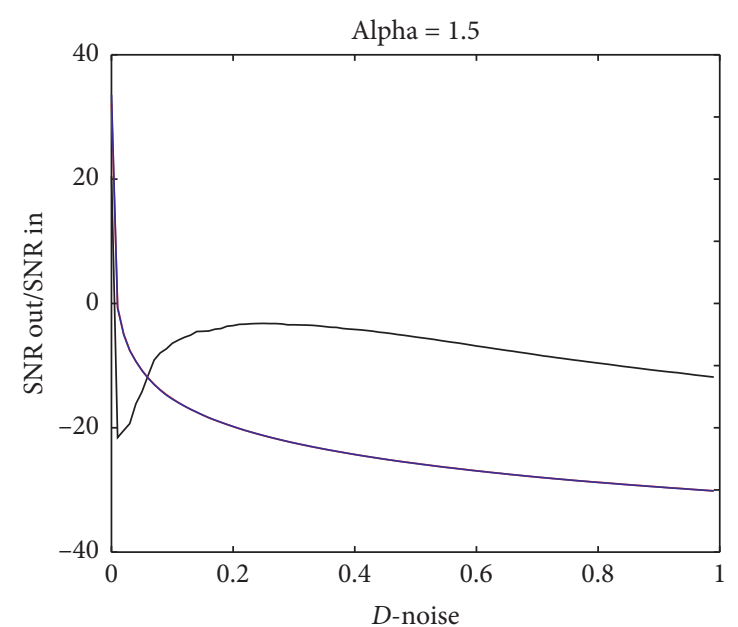

(b)

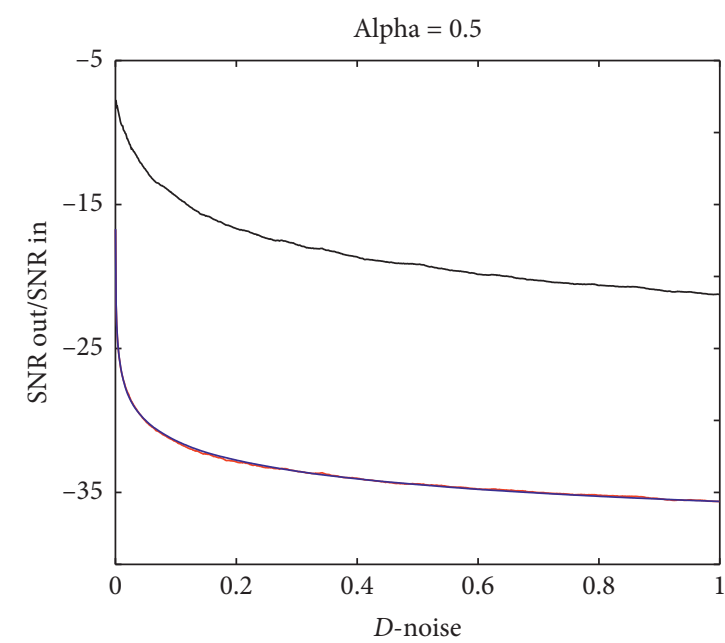

(d)

FIgURE 8: Analysis of SR effect under different noise conditions, $f=10 \mathrm{~Hz}, A=0.1, a=10000$, and $b=4.2 \times e^{12}$. (a) The value of $\alpha$ is 2 . (b) The value of $\alpha$ is 1.5. (c) The value of $\alpha$ is 0.85 . (d) The value of $\alpha$ is 0.5 .

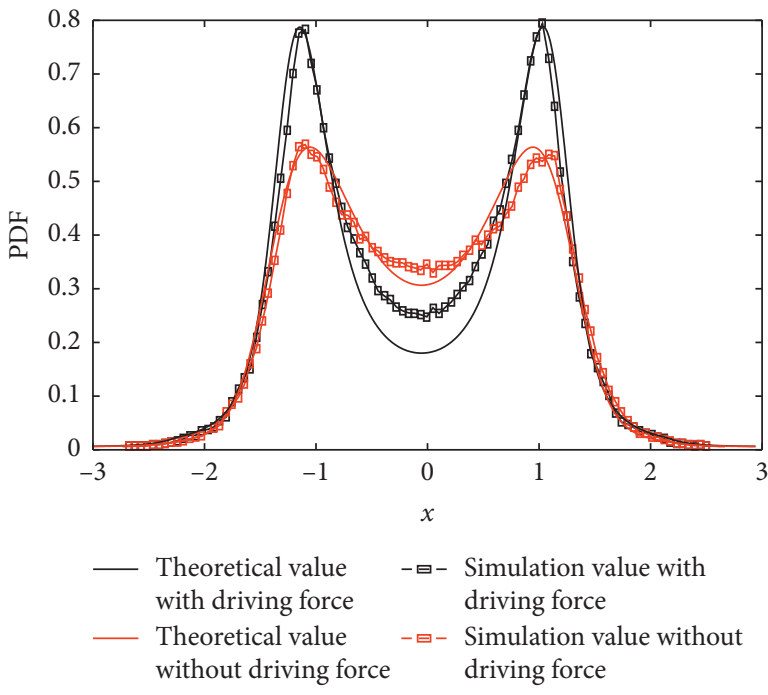

(a)

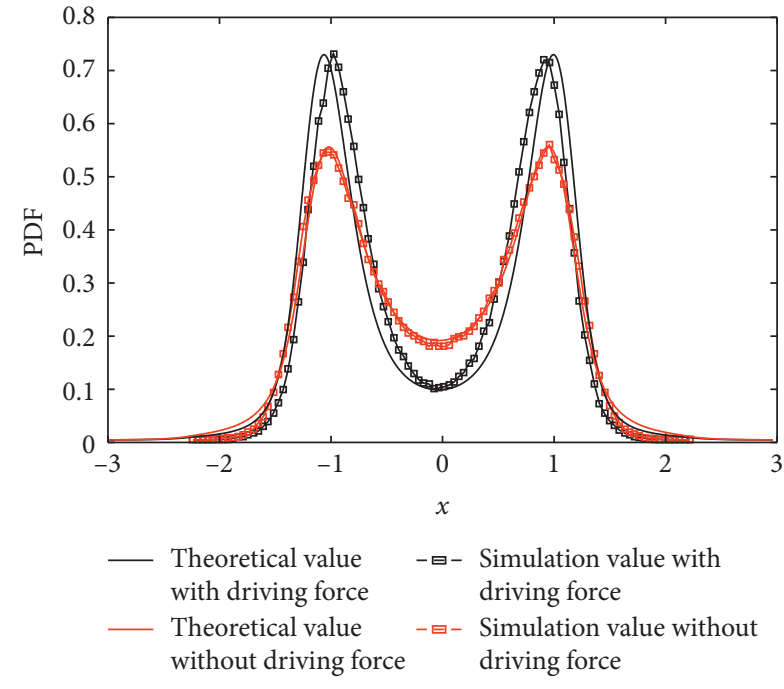

(b)

Figure 9: Continued. 


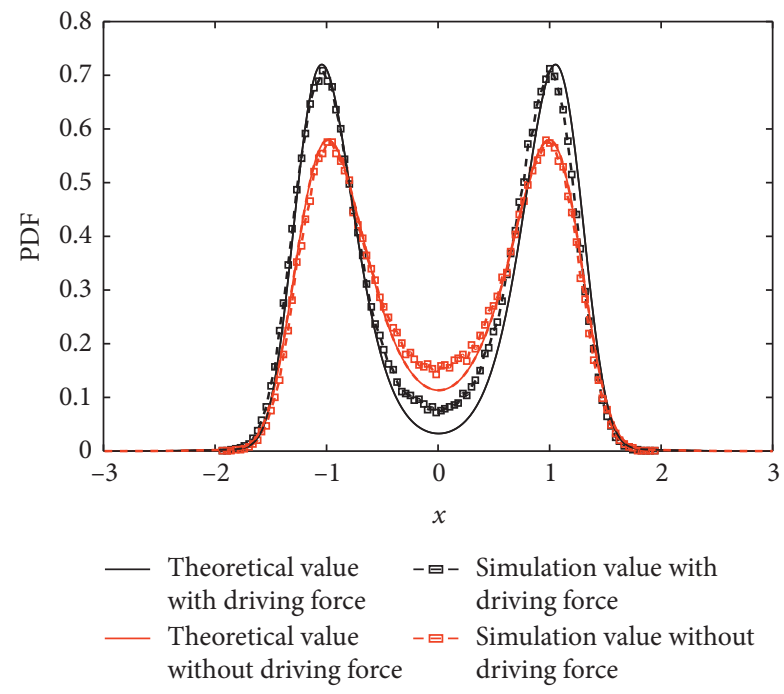

(c)

FIgURE 9: The theoretical and simulation values of PDF after SR processing to the same SNR and a different $\alpha$. (a) The value of $\alpha$ is 0.5 . (b) The value of $\alpha$ is 1.5. (c) The value of $\alpha$ is 2.0.

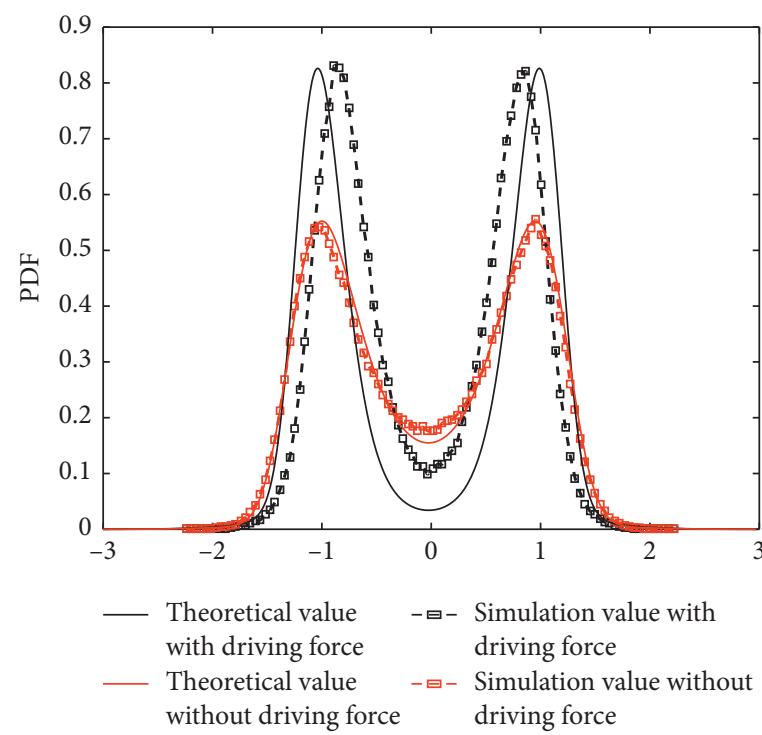

(a)

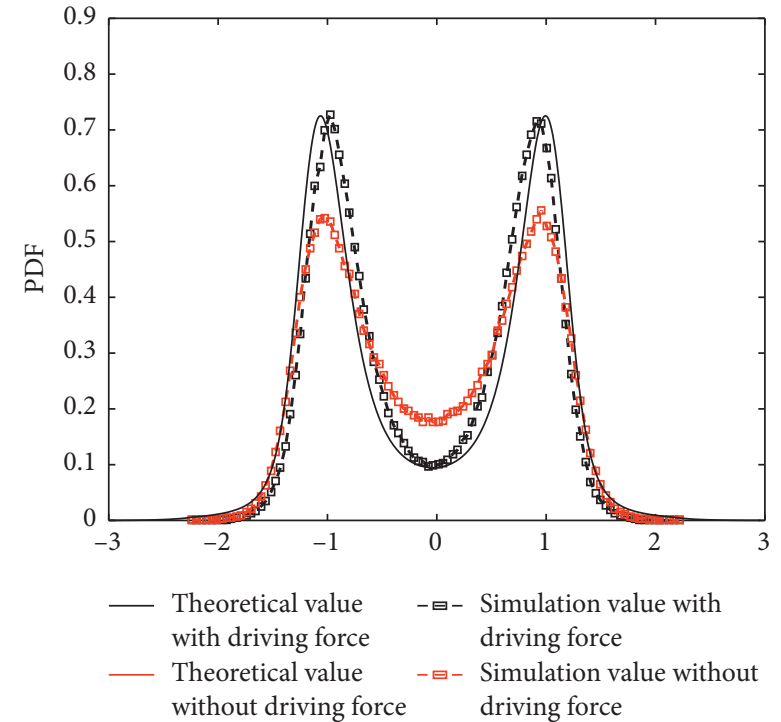

(b)

Figure 10: Continued. 


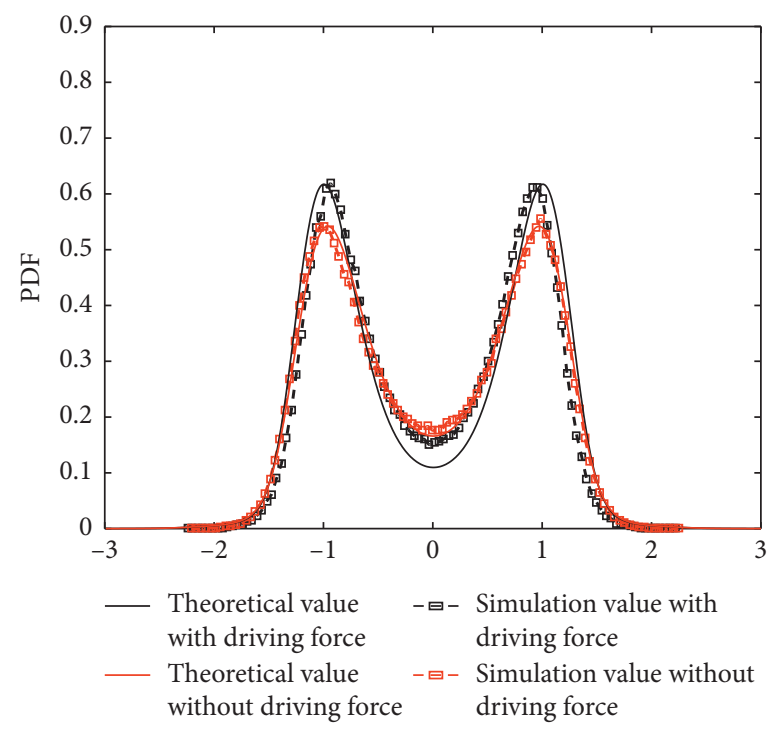

(c)

FIgURE 10: The theoretical and simulation values of PDF after SR processing to the same $\alpha$ and different SNRs. (a) SNR is $8 \mathrm{~dB}$. (b) SNR is $12 \mathrm{~dB}$. (c) SNR is $16 \mathrm{~dB}$.

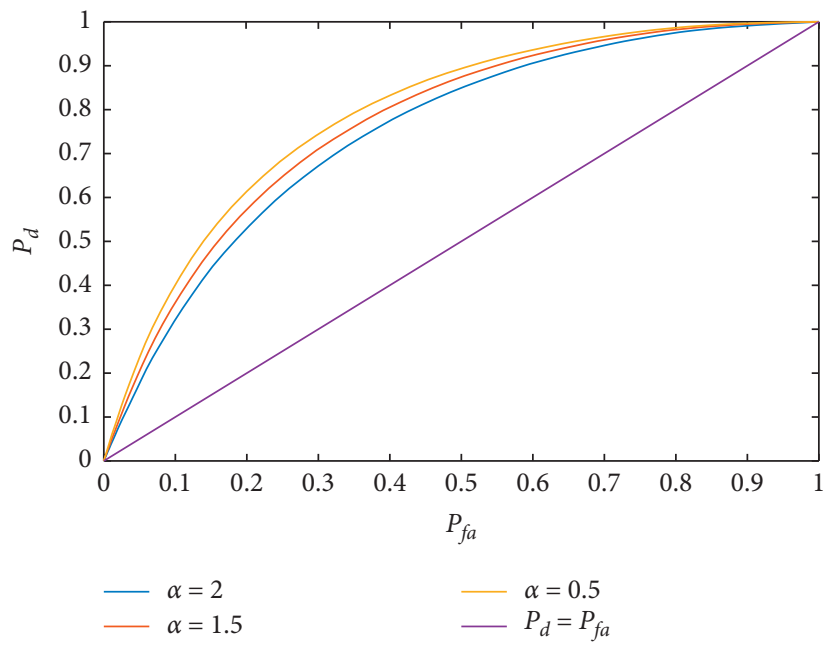

FIGURE 11: ROC curve of DSFH received signal after SR processing under $\alpha$-stable noise (SNR $=-8 \mathrm{~dB})$.

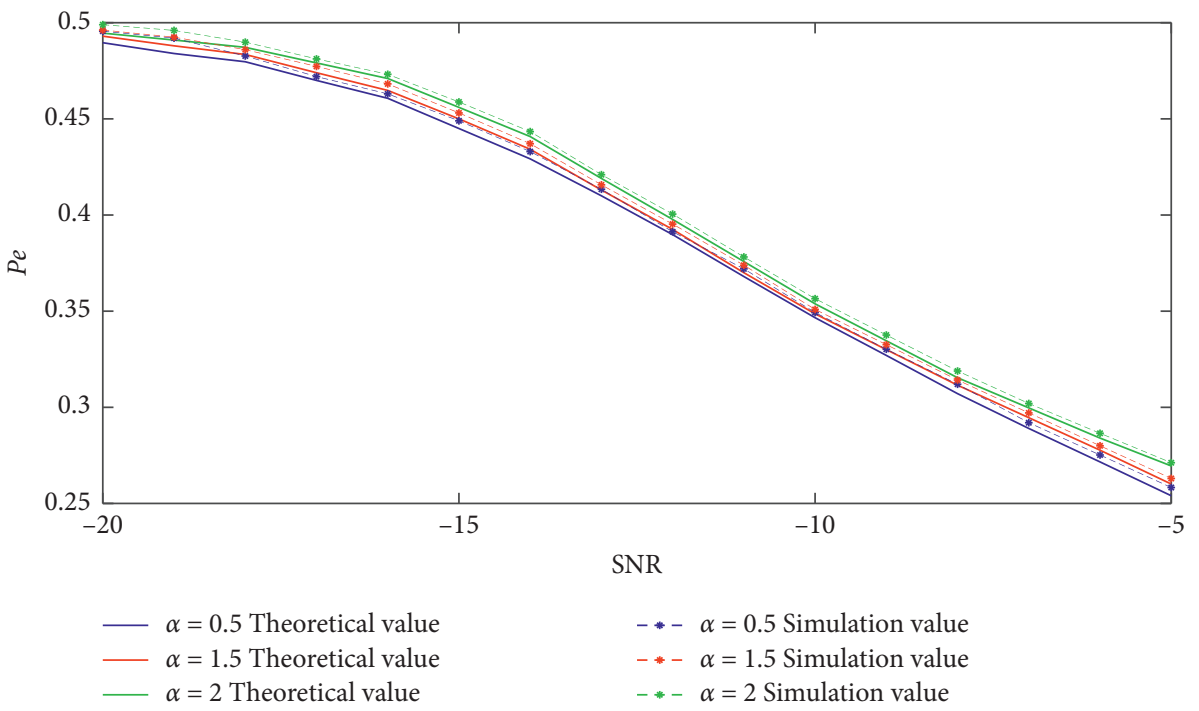

FIGURE 12: BER performance of DSFH received signal processed by SR in $\alpha$-stable noise. 
and processed by SR under $\alpha$-stable noise, and the application range of SNR is further extended to about $-20 \mathrm{~dB}$.

\section{Conclusions}

According to the characteristics of the DSFH signal under $\alpha$-stable noise, the SR method is introduced to detect the sinusoidal signal. Based on the instantaneity of electrical signal transformation, the sampling decision time is used to solve FFPE and the theoretical solution of SR output signal PDF under $\alpha$ stable noise is obtained and verified by simulation. The stages of starting resonance and stable resonance are proposed, and the characteristic is analyzed according to the stage in different noises. Through SNR, noise intensity, and PDF difference analysis, the influence of noise on staring resonance and SNR gain is quantitatively obtained. Noise impulsivity is beneficial to start SR, but it will reduce SNR gain. Based on PDF and MAP criterion, the detection probability and false alarm probability are calculated, and the ROC and Pe-SNR curves are obtained. The availability of the detection method is verified, and the lowest applicable SNR of the system is about $-20 \mathrm{~dB}$. The quantitative conclusion provides the basis for SR to detect and receive DSFH signal under $\alpha$-stable noise and provides a reference for SR application in electromagnetic signal processing. In the future, we will continue to study how to further reduce the BER by designing different receiving structures.

\section{Data Availability}

The data used to support the findings of this study are available from the corresponding or submitting author upon request.

\section{Conflicts of Interest}

The authors declare that there are no conflicts of interest regarding the publication of this paper.

\section{Acknowledgments}

The research was funded by the project "Research on anti jamming technology of dual sequence frequency hopping communication", Natural Science Foundation of Hebei Province of China (Grant nos. F2019506031, F2019506037, and F2020506036), Frontier Innovation Program of Army Engineering University under Grant no. KYSZJQZL2005, Basic Frontier Science and Technology Innovation Program of Army Engineering University under Grant no. KYSZJQZL2020, and Research Innovation Development Funding of Army Engineering University Shijiazhuang Campus under Grant no. KYSZJQZL1902.

\section{References}

[1] F. H. P. Fitzek, "The medium is the message," in Proceedings of the IEEE International Conference on Communications, pp. 5016-5021, Istanbul, Turkey, August 2006.
[2] H. Quan, H. Zhao, and P. Cui, "Anti-jamming frequency hopping system using multiple hopping patterns," Wireless Personal Communications, vol. 81, no. 3, pp. 1159-1176, 2015.

[3] H. Zhao, H. D. Quan, and P. Z. Cui, "Follower-jamming resistible multi-sequence frequency hopping wireless communication system," Systems Engineering and Electronics, vol. 37, no. 3, pp. 671-678, 2015.

[4] Z. Q. Tang, H. D. Quan, and H. X. Sun, “Anti-jamming method of communication based on binary- sequence frequency hopping," Journal of Detection \& Control, vol. 42, no. 1, pp. 56-61, 2020.

[5] Y. B. Wang, H. D. Quan, H. X. Sun, and P. Z. Cui, "Multisequence frequency hopping communication method combined with pseudo-random feature codes," Systems Engineering and Electronics, vol. 42, no. 3, pp. 711-718, 2020.

[6] Y. B. Wang, H. D. Quan, H. X. Sun, and P. Z. Cui, "Antifollower jamming wide gap multi-pattern frequency hopping communication method," Defence Technology, vol. 16, no. 2, pp. 453-459, 2020.

[7] V. Galdi, V. Pierro, and I. M. Pinto, "Evaluation of stochasticresonance-based detectors of weak harmonic signals in additive white Gaussian noise," Physical Review E, vol. 57, no. 6, pp. 6470-6479, 1998.

[8] J. Tang, B. Shi, H. Bao, and Z. Li, "A new method for weak fault feature extraction based on piecewise mixed stochastic resonance," Chinese Journal of Physics, vol. 68, pp. 87-99, 2020.

[9] J. Tang, B. Shi, Z. Li, and Y. Li, "Weak fault feature extraction method based on compound tri-stable stochastic resonance," Chinese Journal of Physics, vol. 66, pp. 50-59, 2020.

[10] Z. Steeve and A. Pierre-Olivier, "On the use of stochastic resonance in sine detection," Signal Processing, vol. 82, no. 3, pp. 353-367, 2002.

[11] R. Gao, "Research on signal detection technology in complex electromagnetic environment," Doctoral Thesis, Xidian University, Xi'an, China, 2015.

[12] L. Liang, "Research on digital signal detection technology based on nonlinear stochastic resonance," Doctoral Thesis, Xidian University, Xi'an, China, 2018.

[13] G. K. Liu, H. D. Quan, H. X. Sun, P. Z. Cui, C. Chi, and S. L. Yao, "Stochastic resonance detection method for the dual-sequence frequency hopping signal under extremely low signal-to-noise radio," Journal of Electronics and Information Technology, vol. 41, no. 10, pp. 2342-2349, 2019.

[14] G.-K. Liu, H.-D. Quan, Y.-M. Kang, H.-X. Sun, P.-Z. Cui, and Y.-M. Han, "A quadratic polynomial receiving scheme for sine signals enhanced by stochastic resonance," Acta Physica Sinica, vol. 68, no. 21, p. 210501, 2019.

[15] B. Mcnamara and K. Wiesenfeld, "Theory of stochastic resonance," Physical Review A, vol. 39, no. 9, pp. 4854-4869, 1989.

[16] G. Hu, Random Forces and Nonlinear Systems, Shanghai Education Press, Shanghai, China, 1994.

[17] X. Q. Hu, Stochastic Resonance Weak Characteristic Signal Detection Theory and Method, National Defense Industry Press, Beijing, China, 2012.

[18] F. B. Duan, "Application of parameter-adjusted stochastic resonance in digital signal transmission," Doctoral Thesis, Zhejiang University, Zhejiang, China, 2002.

[19] E. Field and M. Lewinstein, "Amplitude-probability distribution model for VLF/ELF atmospheric noise," IEEE Transactions on Communications, vol. 26, no. 1, pp. 83-87, 1978. 
[20] J. Omura and P. Shaft, "Modem performance in VLF atmospheric noise," IEEE Transactions on Communication Technology, vol. 19, no. 6, pp. 659-668, 1971.

[21] M. Zimmermann and K. Dostert, "Analysis and modeling of impulsive noise in broad-band powerline communications," IEEE Transactions on Electromagnetic Compatibility, vol. 44, no. 1, pp. 249-258, 2002.

[22] N. H. Nedev, Analysis of the impact of impulse noise in digital subscriber line systems univers, vol. 52, no. 5, , pp. 876-880, University of Edinburgh, Edinburgh, Scotland, 2003, Ph.D. Thesis.

[23] J. Lin, T. Pande, I. H. Kim, A. Batra, and B. L. Evans, "Robust transceiver to combat periodic impulsive noise in narrowband powerline communications," in Proceedings of the IEEE International Conference on Communications, pp. 752-757, London, UK, June 2015.

[24] A. Chechkin, V. Gonchar, J. Klafter, R. Metzler, and L. Tanatarov, "Stationary states of non-linears oscillators driven by Levy noise," Chemical Physics, vol. 284, no. 1-2, pp. 233-251, 2002.

[25] M. D. Paola and G. Failla, "Stochastic response of linear and non-linear system to alpha-stable Levy white noises," Probabilistic Engineering Mechanics, vol. 20, no. 2, pp. 128-135, 2005.

[26] B. Christian, D. M. Alberto, D. P. Mario, and P. Antonina, "First-passage problem for nonlinear systems under Lévy white noise through path integral method," Nonlinear Dynamics, vol. 85, pp. 1445-1456, 2016.

[27] C. A. Natalia and D. P. Mario, "Step-by-step integration for fractional operators," Communications in Nonlinear Science and Numerical Simulation, vol. 59, pp. 292-305, 2018.

[28] B. Dybiec and E. Gudowska-Nowak, "Stochastic resonance: the role of alpha-stable noise," Acta Physica Polonica Seriries B, vol. 37, no. 5, pp. 1479-1490, 2006.

[29] L. Zeng, R. Bao, and B. Xu, "Effects of Lévy noise in aperiodic stochastic resonance," Journal of Physics A: Mathematical and Theoretical, vol. 40, no. 26, pp. 7175-7185, 2007.

[30] W. Y. Zhang, "Research on detection of weak periodic signal in levy noise based on stochastic resonance," Master Thesis, Shanghai Jiaotong University, Shanghai, China, 2009.

[31] J. M. Huang, "Research on stochastic resonance phenomenon of nonlinear system under the background of stable distributed noise," Doctoral Thesis, Zhejiang University, Zhejiang, China, 2012.

[32] G. Zhang, Y. Song, and T. Q. Zhang, "Characteristics analysis of exponential type monostable stochastic resonance under Levy noise," Journal of Electronics \& Information Technology, vol. 39, no. 4, pp. 893-900, 2017.

[33] S. B Jiao, J. Li, Q. Zhang, and G. Xie, "Stochastic resonance in time-delayed asymmetric monostable system with $\alpha$ stable noise," Journal of System Simulation, vol. 28, no. 1, pp. 139146, 2016.

[34] S. B Jiao, D. Sun, J. Li, G. Xie, Y. L. Wu, and Q. Zhang, "Vibrational resonance in a periodic potential system with $\alpha$ stable noise," Acta Physica Sinica, vol. 66, no. 10, p. 100501, 2017.

[35] L. F. He, X. C. Zhou, G. Zhang, and T. Q. Zhang, "Stochastic resonance characteristics analysis of the new potential function under Levy noise and bearing fault detection," Journal of Vibration and Shock, vol. 38, no. 12, pp. 53-62, 2019.

[36] Y. J. Liu, F. Z. Wang, L. Liu, and Y. M. Zhu, "Symmetry tristable stochastic resonance induced by parameter under levy noise background," The European Physical Journal B, vol. 92, p. 168, 2019.

[37] C. Zhang, H. R. Duan, and Y. Xue, "The enhancement of weak bearing fault signatures by stochastic resonance with a novel potential function," Energies, vol. 13, no. 23, pp. 6348-6357, 2020.

[38] Y. J. ZhangDuan, F. Z. Wang, and L. Liu, "Extraction of weak signal in cascaded system under Levy noise," Computer Measurement \& Control, vol. 27, no. 1, pp. 190-194, 2019.

[39] Y. G. Li, Y. Xu, J. Kurths, and X. L. Yue, "Levy-noise-induced transport in a rough triple-well potential," Physical Review E, vol. 94, no. 4-1, p. 042222, 2016.

[40] R.-N. Liu and Y.-M. Kang, "Stochastic resonance in underdamped periodic potential systems with alpha stable Lévy noise," Physics Letters A, vol. 382, no. 25, pp. 1656-1664, 2018.

[41] W. Wang, Z. Yan, and X. B. Liu, "The escape problem and stochastic resonance in a bistable system driven by fractional Gaussian noise," Physics Letters A, vol. 381, no. 29, pp. 2324-2336, 2018.

[42] Y. Liu, J. Liang, S.-B. Jiao, N. Xiao, and M. Hu, "Stochastic resonance of a tri-stable system with $\alpha$ stable noise," Chinese Journal of Physics, vol. 55, no. 2, pp. 355-366, 2017.

[43] D. Han, X. Su, and P. Shi, "Stochastic resonance in multistable system driven by Lévy noise," Chinese Journal of Physics, vol. 56, no. 4, pp. 1559-1569, 2018.

[44] G. L. Zhang, X. L. Lv, and Y. M. Kang, "Parameter-induced stochastic resonance in overdamped system with stable noise," Acta Physica Sinica, vol. 61, no. 4, pp. 1-8, 2012. 